\title{
Graphene Membrane as Suspended Mask for Lithography
}

\author{
Giampiero Amato $\mathbb{D}^{1,2}$ Angelo Greco $\mathbb{D}^{1},{ }^{3}$ and Ettore Vittone $\mathbb{D}^{3}$ \\ ${ }^{1}$ Nanoscience and Materials Division, INRIM, Strada delle Cacce, 91 Torino, Italy \\ ${ }^{2}$ Department of Science and Technological Innovation, University of Eastern Piedmont "A. Avogadro", Viale T. Michel 11, \\ 1512 Alessandria, Italy \\ ${ }^{3}$ Physics Dept. and NIS Interdepartmental Centre, University of Torino, via P. Giuria 1, 10125 Torino, Italy \\ Correspondence should be addressed to Giampiero Amato; g.amato@inrim.it
}

Received 10 June 2018; Revised 4 September 2018; Accepted 13 September 2018; Published 15 October 2018

Guest Editor: Gang Niu

Copyright (C) 2018 Giampiero Amato et al. This is an open access article distributed under the Creative Commons Attribution License, which permits unrestricted use, distribution, and reproduction in any medium, provided the original work is properly cited.

\begin{abstract}
Thanks to its excellent mechanical properties, graphene is particularly suited for the realization of suspended membranes. The present paper deals with one possible application of such membranes that is the realization of suspended lithographic masks for shadow evaporation onto a substrate. This technique, which is largely used for realizing mesoscopic devices, where the quality requirements for the junctions prevent the exposure to ambient air and the occurrence of quantum phenomena requires highly defined structures, can be improved by the use of pure 2-dimensional masks, like graphene ones. Advantages and differences of this material with respect to commonly employed polymers are presented and discussed.
\end{abstract}

\section{Introduction}

The ability to master quantum effects to design and develop new devices, which has led to the emergence of the "second quantum revolution" $[1,2]$, requires the development of new lithographic techniques, suitable for the scaling of the production processes down to the nanoscale, where quantum phenomena become dominant [3].

Among the various techniques contrived aimed at further reducing the scaling of electronic devices, the shadow evaporation technique (or Niemeyer-Dolan technique, NDT) [4,5] offers an effective method to make nanometer-size overlapping structures, which contribute to overcome the limitations of the electron (EBL) [6] or ion (FIB) [7] lithographic techniques, as proximity effects or unwanted ion implantation into the substrate.

Based on the use of a lithographic mask placed not in direct contact with the substrate, the NDT exploits the "shadow" effect during deposition. Actually, the tilting of the substrate at different angles with respect to the atomic source allows thin films to be deposited in spatially distinct or in partially overlapping areas with high spatial resolution, without exposing the sample to the atmosphere and facilitating the fabrication of (hetero) junctions at the nanoscale [8].
Usually, two resist layers with different sensitivities to the electron beam are employed: the lower is generally composed of methyl methacrylate copolymer (MMA), with a greater "sensitivity" to the electron beam than the upper, poly(methyl methacrylate), PMMA. The proximity effects of the beam provoking the polymerization of PMMA have a range on the order of tens of nm, whereas in the MMA, they are found on the hundreds of $\mathrm{nm}$. Then, a broader area of the lower resist will be removed during development so to obtain a geometry with a "substrate" necessary to support the suspended structures defined in the upper resist.

Although the use of such bilayer resist masks is widely adopted to realize the NDT, two main limitations can be identified.

Firstly, the thickness of the suspended layer of PMMA geometrically limits the maximum deposition angle and therefore the reachable portion of the substrate.

Secondly, excessive heating during evaporation can lead to the deformation of the surface of the polymer (the melting point of PMMA is $\sim 110^{\circ} \mathrm{C}$ ), ruining the design of the device and degassing impurities from the mask. This issue occurs, for example, in realizing superconductive mesoscopic devices, which require the deposition of refractory metals (e.g., $\mathrm{Nb}[9]$ or $\mathrm{V}$ [10]); a consequence of the damage of the 
mask is the suppression of superconductivity and the increase in the residual resistance ratio of some types of films [11].

The search for solutions to these problems, therefore, goes towards the replacement of classic polymer resist in favor of new types of $2 \mathrm{D}$ mechanically and thermally more resistant lithographic masks [12], which allow the existing geometric resolution limits to be overcome.

The ultimate 2D mask can be however realized by monolayer materials: graphene has been widely studied and, besides the long list of applications proposed so far [13], its use as a lithographic mask seems immediate, since it has been demonstrated that a monolayer of carbon atoms arranged in the sp2 honeycomb structure is a membrane opaque to other atoms [14]. In fact, even the chemical vapor deposition (CVD) of graphene can be controlled taking advantage from this property, because the first deposited $\mathrm{C}$ layer hinders the reaction of gas precursors with the catalyst underneath [15].

In this perspective, graphene represents a perfect candidate to replace polymers in the manufacture of ultrathin suspended lithographic masks (graphene suspended mask, GSM), presenting the suitable properties of elasticity, mechanical resistance, thermal conductivity, and chemical inertia necessary to solve many of the problems mentioned above. In fact, the reduced intrinsic thickness of graphene ( $<5 \mathrm{~nm}$ for few-layers) considerably increases the limit angle at which thin films can be deposited with the NDT.

The effective creation of the geometries in the mask, occurring through the damage of the layer itself through FIB, might overcome the EBL limit of resolution, the substantial limitation of FIB being related only to the ability in focusing the beam.

In this paper, we demonstrate that few-layer graphene synthesized by CVD on cobalt, possesses adequate mechanical hardness to be employed as a suspended membrane for NDT lithography.

\section{Experimental}

CVD is the production method that offers the largest availability of graphene sheets that can be easily transferred onto a variety of destination substrates. The problem with the largely employed copper-CVD is that the sheets are often discontinuous in correspondence of the $\mathrm{Cu}$ grain boundaries, which is detrimental to both the mechanical and masking properties. Other catalysts, like nickel or cobalt, provide continuous sheets of graphene, because excess of precipitation at the metal grain boundaries keeps the graphene patchwork firmly interconnected [15].

Excellent mechanical stiffness of graphene deposited onto Co has been recently reported [16], which allows to successfully transfer the graphene from the growth substrate without the aid of a strengthening layer (normally, PMMA). This represents a great simplification of the lithographic process, since the polymer, structured with the standard EBL method, is still used as the support of the suspended mask. Removing the additional PMMA layer used for the graphene transfer should remove the support PMMA on the destination substrate, too.
TABLE 1: Summary of the process steps of the CVD growth of graphene on Co. The temperature during the cooling steps has been estimated by a calibrated thermocouple attached to the substrate holder during a dummy deposition process.

\begin{tabular}{lccc}
\hline Process step & $\begin{array}{c}\text { Temperature } \\
\left({ }^{\circ} \mathrm{C}\right)\end{array}$ & $\begin{array}{c}\text { Pressure } \\
(\mathrm{mbar})\end{array}$ & $\begin{array}{c}\text { Gas flow } \\
(\mathrm{sccm})\end{array}$ \\
\hline (i) Heating & $200 \rightarrow 600$ & $100-110$ & $N_{2}=100$ \\
(ii) Heating & $600 \rightarrow 1020$ & $\approx 40$ & $\mathrm{H}_{2}=50$ \\
Annealing + CVD & 1020 & $800-900$ & $\mathrm{H}_{2} / \mathrm{CH}_{4}=50 / 25$ \\
(i) Cooling & $1020 \rightarrow \approx 800$ & $\approx 1.5$ & $\mathrm{~N}_{2} / \mathrm{H}_{2}=100 / 50$ \\
(ii) Cooling & $\approx 800 \rightarrow \approx 200$ & $\approx 1$ & $\mathrm{~N}_{2}=100$ \\
\hline
\end{tabular}

Since the pioneer work of Griffith [17], the mechanical properties of a material are more heavily affected by the amount of defects, rather than the strength of the chemical bonds between the composing atoms. Then, the mechanical properties of a material with reduced dimensionality are expected to improve with respect to its bulk version, because in that case, it is in principle easier to reduce the percentage of defects. According to the Raman analysis reported in [16], the defect-related peak at $\sim 1350 \mathrm{~cm}^{-1}$ is often below the detection limit in wide maps of graphene grown on Co.

When the number of atomic layers varies, the graphene decreases its tensile strength and becomes more "fragile," but it has been reported that this variation is of the order of few percent up to 7 layers [18]. Then, the eventual presence of multilayered regions in graphene used for the lithographic process is the best compromise between the need of reducing both uncontrolled discontinuities in the mask and the supporting PMMA during transfer and the need of having an ultrathin mask. A multilayered graphene mask is still 2 orders of magnitude thinner than the corresponding traditional polymer mask.

Co films, $250-300 \mathrm{~nm}$ thick, thermally evaporated onto oxidized Si substrates have been employed for the CVD of graphene. This has been carried out in a quartz furnace OTF-1200X from MTI Corporation with the process steps listed in Table 1.

Pressure is close to atmospheric during the deposition. This is obtained by manually closing the valve between the deposition chamber and the primary pump so as to reduce the conductance of the process gas discharge pipe and the pumping speed of the system. Increasing the amount of gas in the chamber speeds up the catalysis and reduces the deposition step to $5 \mathrm{~min}$, counteracting in this way the occurrence of dewetting of Co [19].

The cooling of the cobalt substrate triggers the process of carbon precipitation on the surface of the film, leading to the formation of graphene multilayer. The control of the cooling time allows the control of the amount of precipitation, which then determines the thickness of the graphene layer. Flash cooling is achieved by physically moving the sample from the hot zone of the furnace to an area approximately at room temperature. The sample temperature decreases in an exponential way, with a time constant of about $300 \mathrm{~s}$.

Samples have been characterized by Raman spectroscopy, either on the growth substrate [19] or after transfer 


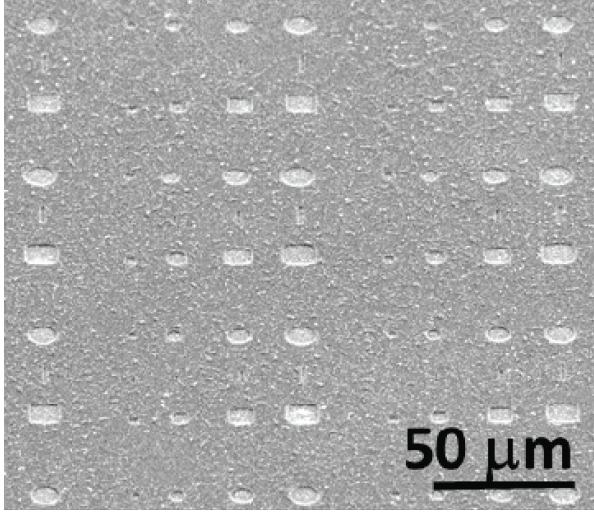

(a)

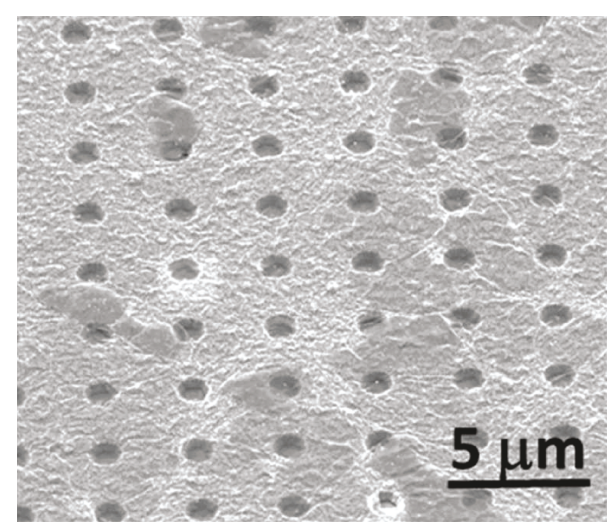

(b)

FIGURE 1: SEM images of graphene transferred to submasks in PMMA (a) and Ti (b).

onto oxidized Si. Spectra display an intensity ratio between the $2 \mathrm{D}$ mode at $\sim 2700 \mathrm{~cm}^{-1}$ and the $\mathrm{G}$ mode at $\sim 1600 \mathrm{~cm}^{-1}$ around $1 / 2$, suggesting the multilayer character of graphene. The $\mathrm{D}$ mode, related to defects, is practically absent, as mentioned before.

Suspended graphene masks require support structures ("submasks"). These were made of polymer or $\mathrm{Ti}$, as detailed hereafter:

(i) Polymer submask: composed of a double layer of copolymer (MMA) and polymer (PMMA A4), obtained on a $\mathrm{SiO}_{2}$ substrate by double deposition of PMMA and MMA necessary for achieving the desired interspace with the substrate. The thickness of the MMA (PMMA) layer was measured to be $\sim 667(\sim 241) \mathrm{nm}$ by ellipsometry, resulting in an interspace $\sim 908 \mathrm{~nm}$. Circular areas with $1-4 \mu \mathrm{m}$ diameter have been exposed to the electron beam and then removed (Figure 1(a))

(ii) $\mathrm{Ti}$ submask: this particular submask has been designed to be compatible with deposition processes in which the mask is exposed to high temperatures. Apart from the deposition of refractory metals described above, this kind of mask is needed when the deposition occurs onto a substrate kept at high $T$, as in the case of MBE. Here, EBL was used similarly to the previous case. A structure of the polymer made up of pillars was used as a mask for depositing the Ti layer with film thickness of $300 \mathrm{~nm}$. The resulting lift-off of the polymer pillars created the circular hole structure visible in Figure 1(b)

The transfer of graphene to the support structures is done by the well-known "picking-up" method without the need of a strengthening PMMA layer onto graphene [16].

The interspace with the substrate is then controlled by choosing an appropriate thickness for the submask. Here, a limitation for the Ti mask is due to the difficulty of growing very thick metal films. Then, during drying, capillary forces take place at the meniscus of the liquid trapped in the substrate-membrane cavity. These forces are inversely

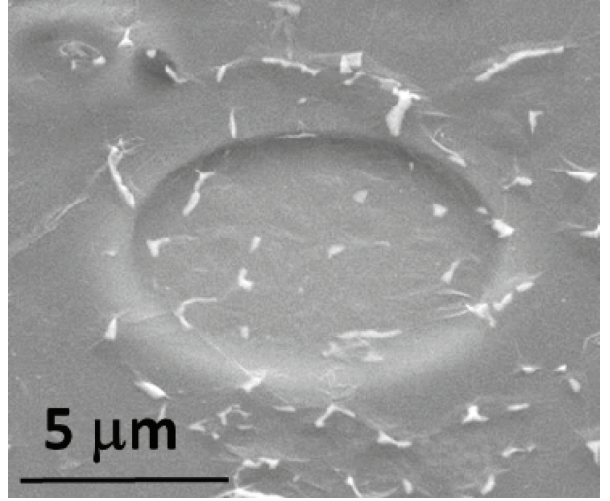

FIgURE 2: SEM micrograph of a membrane suspended on PMMA submask.

proportional to the interspace distance and linearly dependent to the area of the hole [20]. As a consequence, we observed a percentage of membranes collapsed during drying, statistically higher for larger holes and for smaller interspace distances. As a matter of fact, graphene membranes survived only onto smaller holes in the case of $\mathrm{Ti}$ $(\sim 300 \mathrm{~nm})$ submasks.

At present, we have not identified the mechanism of liquid evaporation out of the cavities: actually, after the graphene has been laid down, it should seal the cavity. To reduce the mortality of membranes during drying, attempts using low surface tension liquids, like pentane, which has been successfully employed in the drying of porous materials [21], are underway. This in is particular for metal submasks, because the interspace is thinner than polymer submask, which, on the opposite, can be damaged by pentane.

As shown in Figure 2 the graphene sheet excellently adheres to the substrate. What is obtained from this process are graphene suspended membranes on which the graphene suspended mask of any shape can be realized by FIB.

Openings in the membrane must be done in order to predict the geometry of the film that is to be deposited, also considering the deposition angle. The "carving" of these geometries on GSM was realized with a milling process, using 


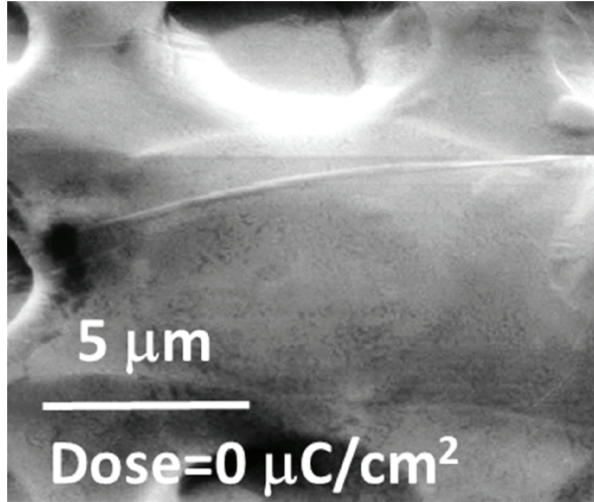

(a)

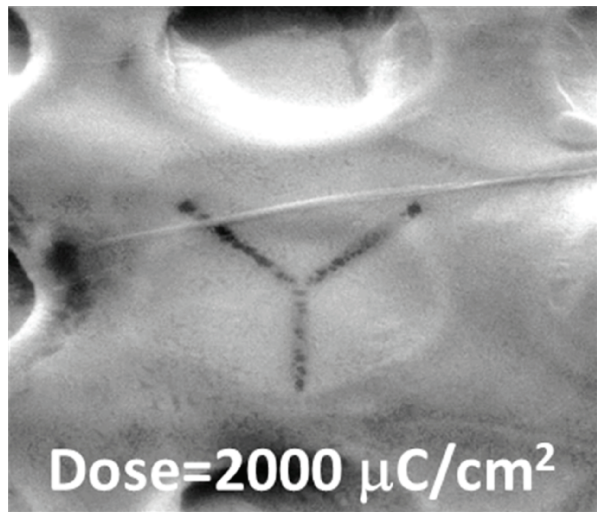

(c)

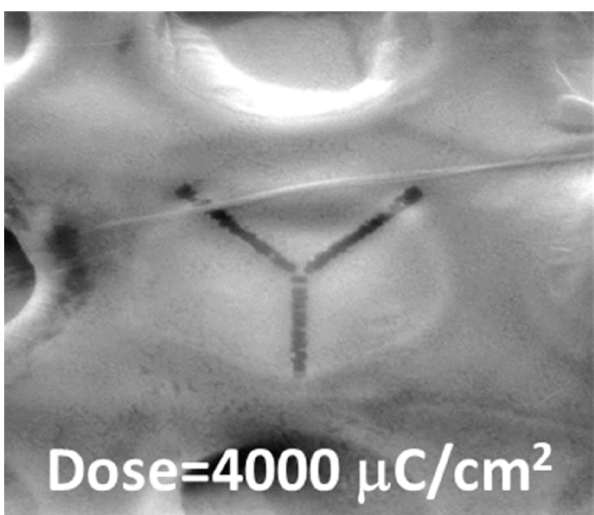

(e)

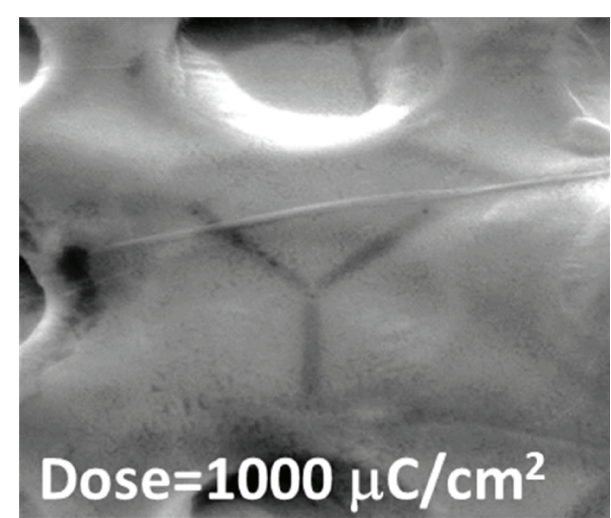

(b)

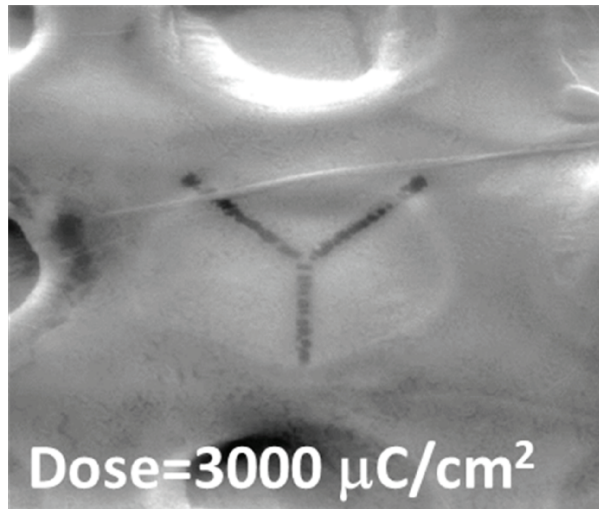

(d)

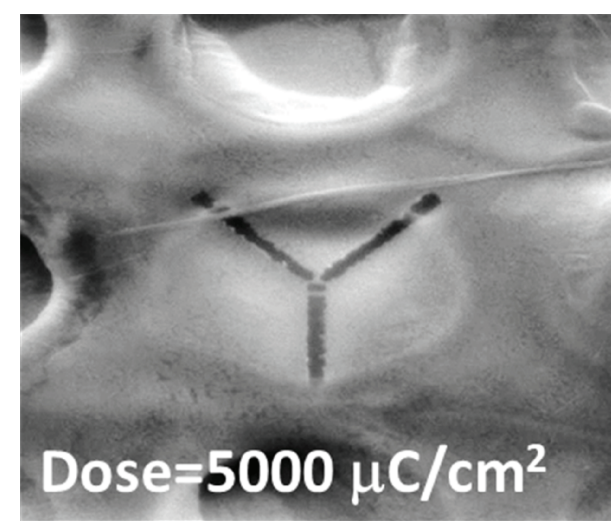

(f)

Figure 3: From (a) to (f): effects on the irradiation of the membrane by a dose increasing by steps of $1000 \mu \mathrm{C} / \mathrm{cm}^{2}$.

$\mathrm{Ga}$ ions with an energy of $30 \mathrm{keV}$. To carve the graphene without implanting excess $\mathrm{Ga}$ ions into the substrate, we performed a "dose test," in order to determine the minimum level of irradiation needed to delineate the desired geometry. For this test, irradiation was carried out on an area of $\sim 4.5 \cdot 10^{-8} \mathrm{~cm}^{2}$ using an ion beam current $I=9.8 \mathrm{pA}$. This is the lowest possible current available in the FIB column, which was chosen to have the maximum control of the dose. Figure 3 shows different situations, as achieved by increasing the dose of $1000 \mu \mathrm{C} / \mathrm{cm}^{2}$ from one step to the other until the layer is completely broken. As displayed by the successive micrographs of Figure 3, there is no definite threshold at which the carving of graphene takes place, rather a progressive erosion of the membrane is observed. This does not help to minimize the dose of $\mathrm{Ga}$ ions that can further implant into the substrate. This aspect can be detrimental to the correct behavior of devices, and then a possible solution can be to deposit the active layers at different tilting angles so to use a different region of the substrate.

The optimal dose for the incision of the mask shown in Figure 3 was estimated to be $5000 \mu \mathrm{C} / \mathrm{cm}^{2}$. It is important to report that the single dose of $1000 \mu \mathrm{C} / \mathrm{cm}^{2}$ was provided over the entire area by means of successive irradiation of 500 doses since it was noted that a gradual irradiation causes less stress on the membrane allowing better resolution in most geometries. A better focusing of the ion beam allows 


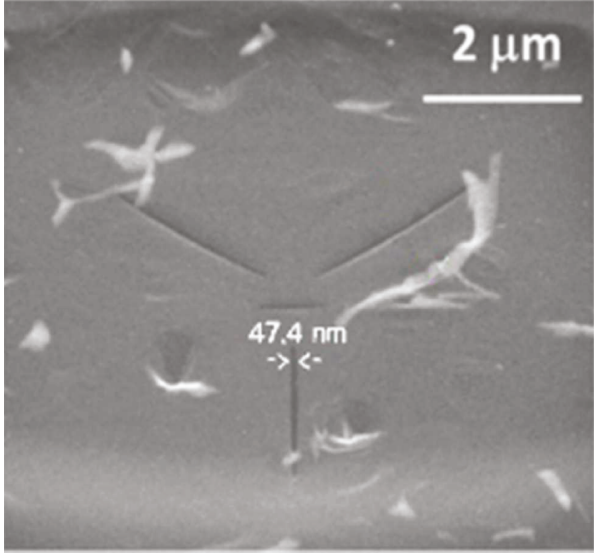

(a)

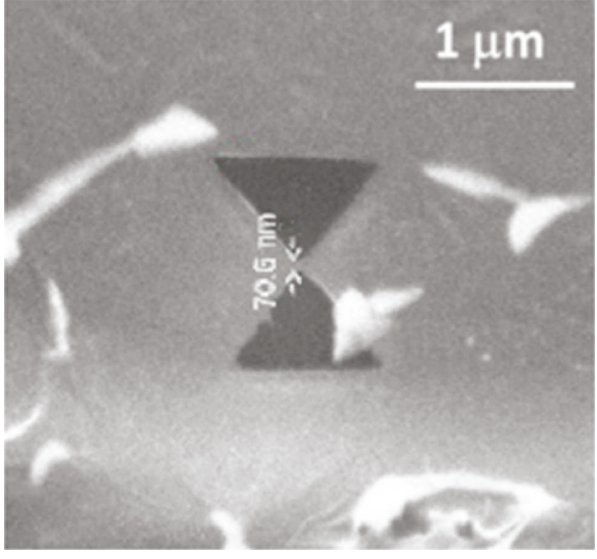

(b)

FIGURE 4: SEM micrographs on suspended membranes carved by FIB with a dose of $5000 \mu \mathrm{C} / \mathrm{cm}^{2}$. In the photograph on (b), we measured the radius of a circle inscribed in the corner of the engraved triangle obtaining a value of $\sim 19 \mathrm{~nm}$, hardly obtainable with the conventional polymeric masks.

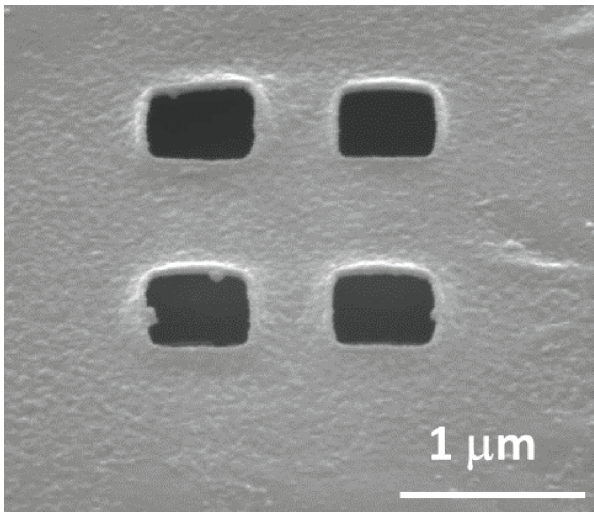

(a)

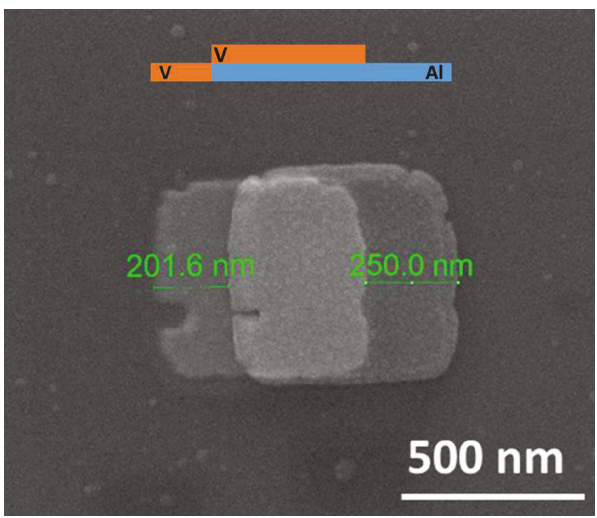

(b)

FIGURE 5: (a) SEM image of the four rectangular holes engraved on a GSM, to measure the shift of the deposited pads with the tilting angle: the tilt is along the horizontal side of the $500 \times 500 \mathrm{~nm}^{2}$ squares. (b) The shift measurements between the first (right, $\mathrm{Al}$ ) and the second (left, $\mathrm{V}$ ) depositions for the squared geometry (on top the scheme of the deposition).

the realization of geometries with linear dimensions in the order of $10 \mathrm{~nm}$, as it is visible in Figure 4, which shows strips and bridges with widths of the order of $50 \mathrm{~nm}$.

\section{Results and Discussion}

The deposition tests were carried out using the NDT to evaluate the behavior of the suspended masks during a double-angled deposition by means of an electron beam apparatus. Simple geometries were chosen, such as squares and circles, to estimate the shadowing effect of the mask on elementary shapes.

The double-angled deposition of $\mathrm{Al}$ and $\mathrm{V}$ on PMMA submask, 908 nm thick, is shown in Figure 5. At first, a $22 \mathrm{~nm}$ thick Al layer was deposited with an angle of $0^{\circ}$ with respect to the vertical, at a rate of $0.1 \mathrm{~nm} / \mathrm{s}$; therefore, $\mathrm{V}$ was deposited at an angle of $19^{\circ}$ from the vertical, with a thickness of $22 \mathrm{~nm}$ at a rate of $0.2 \mathrm{~nm} / \mathrm{s}$.
These metals have been chosen because they are used for hybrid single electron transistors (SET) [22]. Moreover, V is a refractory material (melting temperature $\sim 1910^{\circ} \mathrm{C}$ ), then it is interesting to test the behavior of GSM to an intense heat irradiation during the deposition step.

The shift $\Delta x$ between patterns deposited with an angle difference of $\Delta \theta$ is predicted through the simple relationship $\Delta x=d \cdot \tan \theta$, where $d$ is the mask interspace. Considering $d \sim 900 \mathrm{~nm}$ and $\Delta \theta=19^{\circ}, \Delta x \sim 313 \mathrm{~nm}$, a value that has not been observed (Figure 5).

On the contrary, it can be observed that the second $\mathrm{V}$ deposition did not affect the integrity of the GSM.

The shift, as shown in Figure 5, is also different depending on the side of observation. The difference is of $\sim 50 \mathrm{~nm}$. It is also observed that the dimension of the patterns deposited at different angles is not the same since the linear dimensions of the geometries from the first to the second deposition are reduced by $\sim 50 \mathrm{~nm}$. While the dimension 


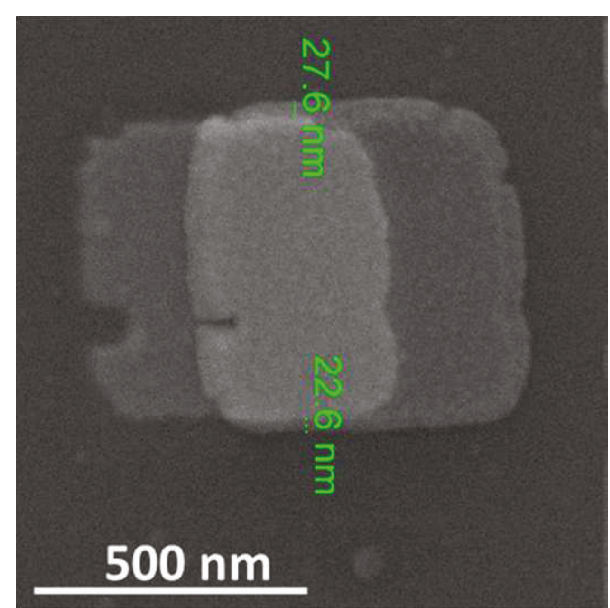

(a)

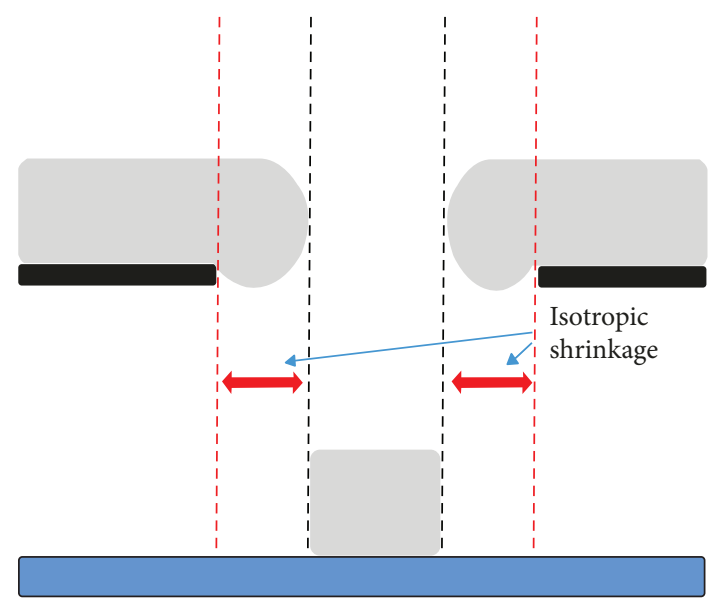

(b)

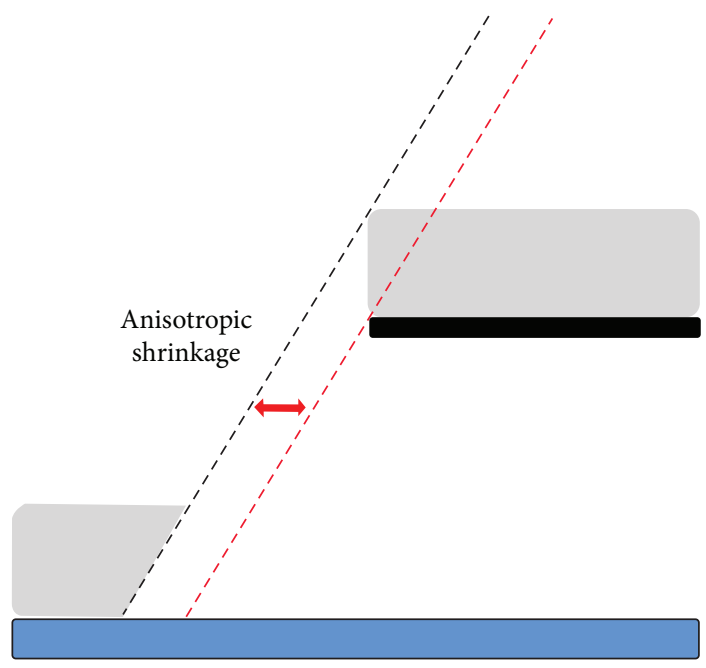

(c)

FIGURE 6: (a) SEM image evidencing the narrowing of the geometries from the first (Al, right) to the second (V, left) deposition, affecting the vertical side, which are not affected by tilting on the pattern of Figure 5. (b) and (c) display the isotropic shrinkage of the mask due to deposition of metal at the edges and the anisotropic shrinkage related to the increase of the thickness of the mask, respectively.

along the horizontal axis of the two micrographs is affected by the angle of tilt, the narrowing along the vertical axis can be related to a shrinking effect of the mask consequent to the first deposition, as shown in Figure 6.

Since this isotropic shrinkage effect of the openings in the masks is very close to the thickness of the material deposited during the first deposition ( $25 \mathrm{~nm}$ per side, Figure 6(a)), we can argue that the metal deposits on edges of the suspended membrane induce a progressive occlusion of the mask with the thickening of the thin film (Figure 6(b)). This effect is generally negligible in masks suspended in PMMA. On the other hand, using a GSM, this phenomenon must be taken into consideration, since the two-dimensional membrane acquires a thickness that is no longer negligible if compared to the initial thickness. In addition, the difference in the shift with respect to the trigonometric calculation can be explained keeping into account the 3-dimensionalization of the membrane (Figure 6(c)).

However, the changes in the mask geometry, due to the deposition of the first layer of metal, can be quite easily

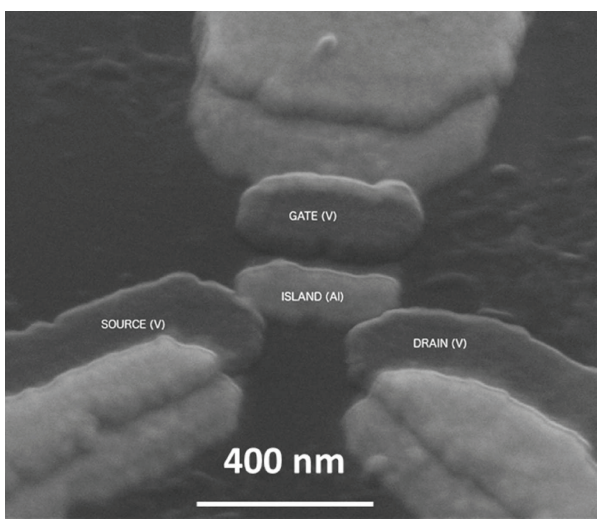

FIgURE 7: Micrograph of a hybrid, SNS single electron transistor, realized by a double-angled evaporation of $\mathrm{Al}$ and $\mathrm{V}$ through a GSM.

predicted. As an example, the anisotropic shrinkage displayed in Figure 6(c) can be circumvented simply by carrying out the second evaporation at $0^{\circ}$, inversely to what has been 


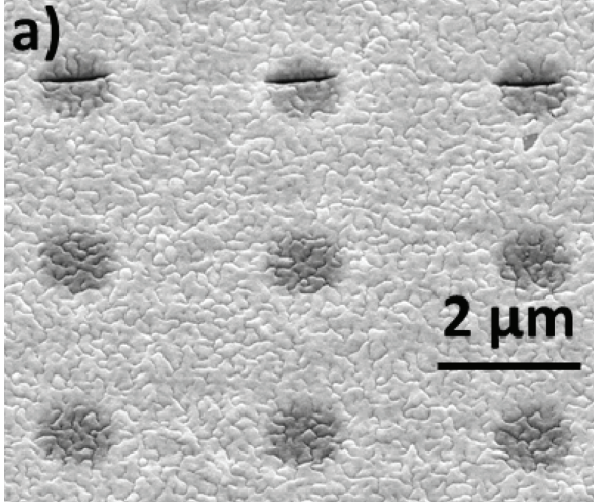

(a)

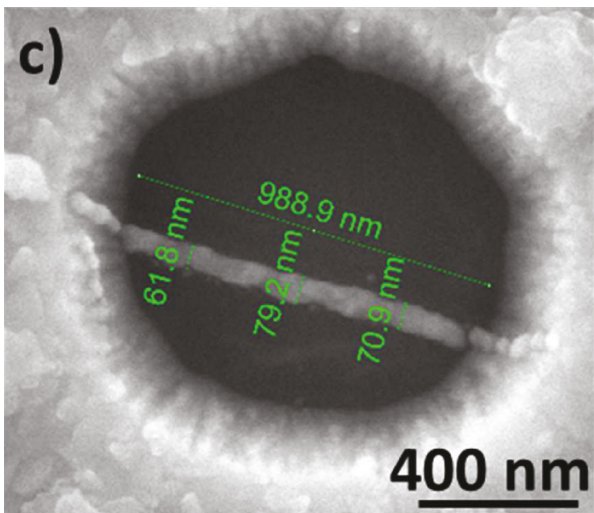

(c)

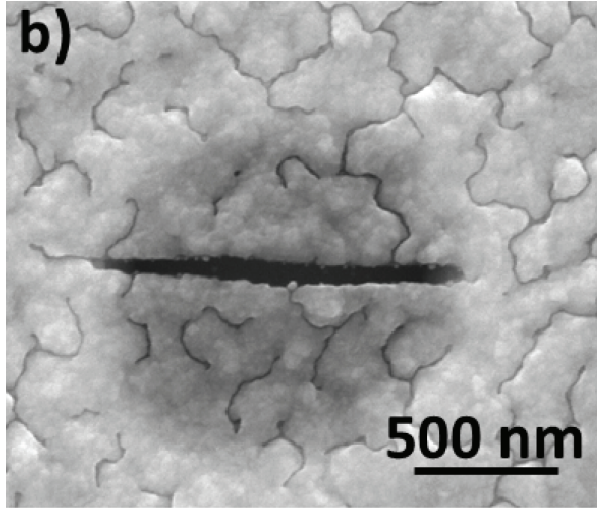

(b)

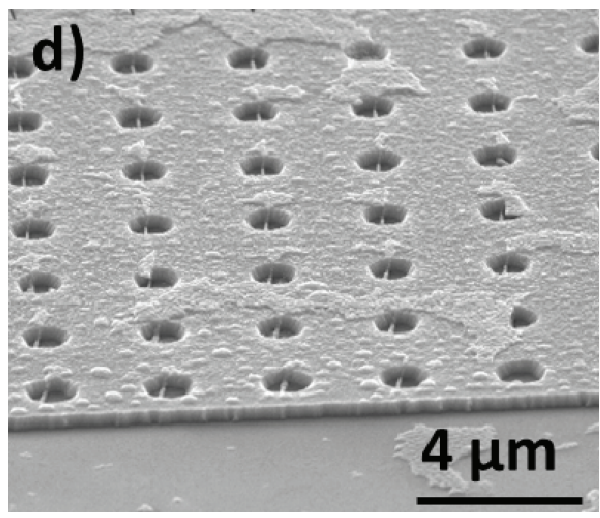

(d)

Figure 8: (a)SEM micrographs after Ge deposition on GSM with intact (bottom 6) and carved (top 3) structures; (b) a close view of a carved membrane with Ge on top; (c) dimensional measurements on the Ge nanowire after mask lift-off; (d) an array of wires.

previously done. It is then possible to realize devices like the hybrid SET shown in Figure 7. In this case, the SNS assembly is achieved by depositing superconducting $(\mathrm{V})$ source and drain [22] and a normal metal ( $\mathrm{Al}$ in the case of operating $\mathrm{T}$ higher than the critical $\mathrm{T}$ of $\mathrm{Al}$ ) for the island [23]. The tunnel junctions between the source (drain) with the island are realized by oxidizing the $\mathrm{Al}$ deposited at the first evaporation step. Typically, exposing the island to 1 bar of $\mathrm{O}_{2}$ provides tunnel resistance values of the order of $10^{4}-10^{5} \Omega$. The flexibility of the NDT allows to easily include the oxidation step between the two depositions, without breaking the vacuum [23]. The gate is capacitively coupled with the island, the second capacitor armature being the second evaporation through the opening used for the island itself. The characterization of the devices obtained by means of GSM is underway.

As the last application of GSM, we carried out a deposition in typical MBE conditions. In this case, the substrate is generally heated to temperatures exceeding some hundred ${ }^{\circ} \mathrm{C}$, in order to enhance the surface atom mobility, facilitating in this way the crystalline arrangement of the deposit. Polymeric masks do not withstand these conditions, so we used a GSM lying onto a Ti submask. Using FIB, linear slits $\sim 70 \mathrm{~nm}$ wide and with length equal to the diameter of the suspended membranes were opened in graphene lying onto circular holes with diameter of $\sim 1 \mu \mathrm{m}$ and thickness of $300 \mathrm{~nm}$ realized on a Ti film on a $\mathrm{SiO}_{2}$ substrate.
A thin film of germanium was then deposited with a total thickness of $40 \mathrm{~nm}$ at the rate of $\sim 4 \AA / \mathrm{min}$. During the deposition, the pressure in the chamber was kept at $P=1.2 \cdot 10^{-9}$ mbar and the substrate temperature at $T \sim 400^{\circ} \mathrm{C}$. Subsequently, a lift-off was carried out by exfoliating the graphene with the upper Ge layer covering the whole sample (Figure 8). Obviously, the present experiment cannot be correctly defined as an MBE growth, rather as a simulation of $M B E$ growth. Actually, the presence of the Ti submask and the graphene on top prevents the standard chemical cleaning procedure of the substrate so to favor the crystalline growth. Being the substrate covered by amorphous $\mathrm{SiO}_{2}$, the $\mathrm{Ge}$ nanowire has a polycrystalline structure. Nevertheless, here, the aim was to demonstrate the reliability of the mask at these harsh conditions. The definition of a preparation protocol, compatible with MBE requirements, is under study.

\section{Conclusions}

This report shows the potential of GSM, which can offer remarkable advantages with respect to traditional polymeric masks. The shadow evaporation technique allowed depositing structures with high definition without any apparent damage of the graphene layer, even when the target is subjected to heating. Disadvantages are related to the possible implant of foreign atoms in the substrate, as provided by 
the FIB used to carve the mask and to the low reliability of the wet technique employed to transfer graphene onto the submask. In both cases, however, remedies seem at hand, namely, the angled carving of the membrane, so to implant ions in unused regions of the substrate, and the use of solvents with low surface tension. Another phenomenon, say, the progressive 3-dimensionalization of the mask during evaporation, can be easily predicted, too. Surprisingly, we also observed a lateral growth of the metal film at graphene edges, which is responsible for a reduction of the pattern size with time. Since this reduction is closely related to the amount of evaporated metal, this phenomenon can be easily controlled and used for particular purposes, e.g., as a tool for further improvement of the resolution of the technique.

\section{Data Availability}

The data used to support the findings of this study are available from the corresponding author upon request.

\section{Conflicts of Interest}

The authors declare that they have no conflicts of interest.

\section{Acknowledgments}

EV acknowledges support from the project "Departments of Excellence" (L. 232/2016), funded by the Italian Ministry of Education, University and Research (MIUR).

\section{References}

[1] J. P. Dowling and G. J. Milburn, "Quantum technology: the second quantum revolution," Philosophical Transactions of the Royal Society A: Mathematical, Physical and Engineering Sciences, vol. 361, no. 1809, pp. 1655-1674, 2003.

[2] J. Jones, "The second quantum revolution," Physics World, vol. 26, no. 08, pp. 40-41, 2013.

[3] L. L. Sohn, "Nanotechnology: a quantum leap for electronics," Nature, vol. 394, no. 6689, pp. 131-132, 1998.

[4] G. J. Dolan, "Offset masks for lift-off photoprocessing," Applied Physics Letters, vol. 31, no. 5, pp. 337-339, 1977.

[5] J. Niemeyer and V. Kose, "Observation of large DC supercurrents at nonzero voltages in Josephson tunnel junctions," Applied Physics Letters, vol. 29, no. 6, pp. 380-382, 1976.

[6] A. N. Broers, A. C. F. Hoole, and J. M. Ryan, "Electron beam lithography-resolution limits," Microelectronic Engineering, vol. 32, no. 1-4, pp. 131-142, 1996.

[7] J. Orloff, "High resolution focused ion beams," Review of Scientific Instruments, vol. 64, no. 5, pp. 1105-1130, 1993.

[8] H. Pothier, P. Lafarge, P. F. Orfila, C. Urbina, D. Esteve, and M. H. Devoret, "Single electron pump fabricated with ultrasmall normal tunnel junctions," Physica B: Condensed Matter, vol. 169, no. 1-4, pp. 573-574, 1991.

[9] M. Peiniger and H. Piel, "A superconducting Nb3Sn coated multicell accelerating cavity," IEEE Transactions on Nuclear Science, vol. 32, no. 5, article 3610, 3612 pages, 1985.

[10] N. E. Alekseevskii, V. M. Sakosarenko, K. Blüthner, and H.-J. Köhler, "Superconducting properties of vanadium films," Physica Status Solidi (A), vol. 34, no. 2, pp. 541-546, 1976.
[11] T. Henning, "Charging effects in niobium nanostructures," http://arxiv.org/abs/cond-mat/9901308.

[12] M. Sekimoto, H. Yoshihara, and T. Ohkubo, "Silicon nitride single-layer X-ray mask," Journal of Vacuum Science and Technology, vol. 21, no. 4, pp. 1017-1021, 1982.

[13] A. C. Ferrari, F. Bonaccorso, V. Fal'ko et al., "Science and technology roadmap for graphene, related two-dimensional crystals, and hybrid systems," Nanoscale, vol. 7, no. 11, pp. 4598-4810, 2015.

[14] V. Berry, "Impermeability of graphene and its applications," Carbon, vol. 62, pp. 1-10, 2013.

[15] C. Mattevi, H. Kim, and M. Chhowalla, "A review of chemical vapour deposition of graphene on copper," Journal of Materials Chemistry, vol. 21, no. 10, pp. 3324-3334, 2011.

[16] G. Amato, F. Beccaria, and F. Celegato, "Growth of strained, but stable, graphene on Co," Thin Solid Films, vol. 638, pp. 324-331, 2017.

[17] A. A. Griffith, "The phenomena of rupture and flow in solids," Philosophical Transactions of the Royal Society A: Mathematical, Physical and Engineering Sciences, vol. 221, no. 582-593, pp. 163-198, 1921.

[18] Y. Y. Zhang and Y. T. Gu, "Mechanical properties of graphene: effects of layer number, temperature and isotope," Computational Materials Science, vol. 71, no. 1, pp. 197-200, 2013.

[19] G. Amato, "High temperature growth of graphene from cobalt volume: effect on structural properties," Materials, vol. 11, no. 2, p. 257, 2018.

[20] D. Chandra and S. Yang, "Stability of high-aspect-ratio micropillar arrays against adhesive and capillary forces," Accounts of Chemical Research, vol. 43, no. 8, pp. 1080-1091, 2010.

[21] O. Belmont, D. Bellet, and Y. Bréchet, "Study of the cracking of highly porous $\mathrm{p}^{+}$type silicon during drying," Journal of Applied Physics, vol. 79, no. 10, article 7586, 7591 pages, 1996.

[22] C. P. Garcia and F. Giazotto, "Josephson current in nanofabricated $\mathrm{V} / \mathrm{Cu} / \mathrm{V}$ mesoscopic junctions," Applied Physics Letters, vol. 94, no. 13, article 132508, 2009.

[23] G. Amato and E. Enrico, "Current status and technological limitations of hybrid superconducting-normal single electron transistors," in Superconductivity-Theory and Applications, A. Luiz, Ed., InTech, 2011. 


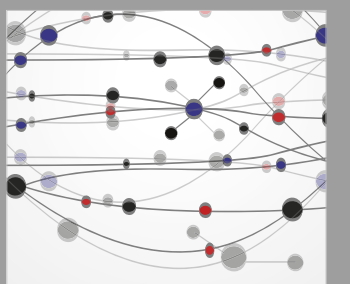

The Scientific World Journal
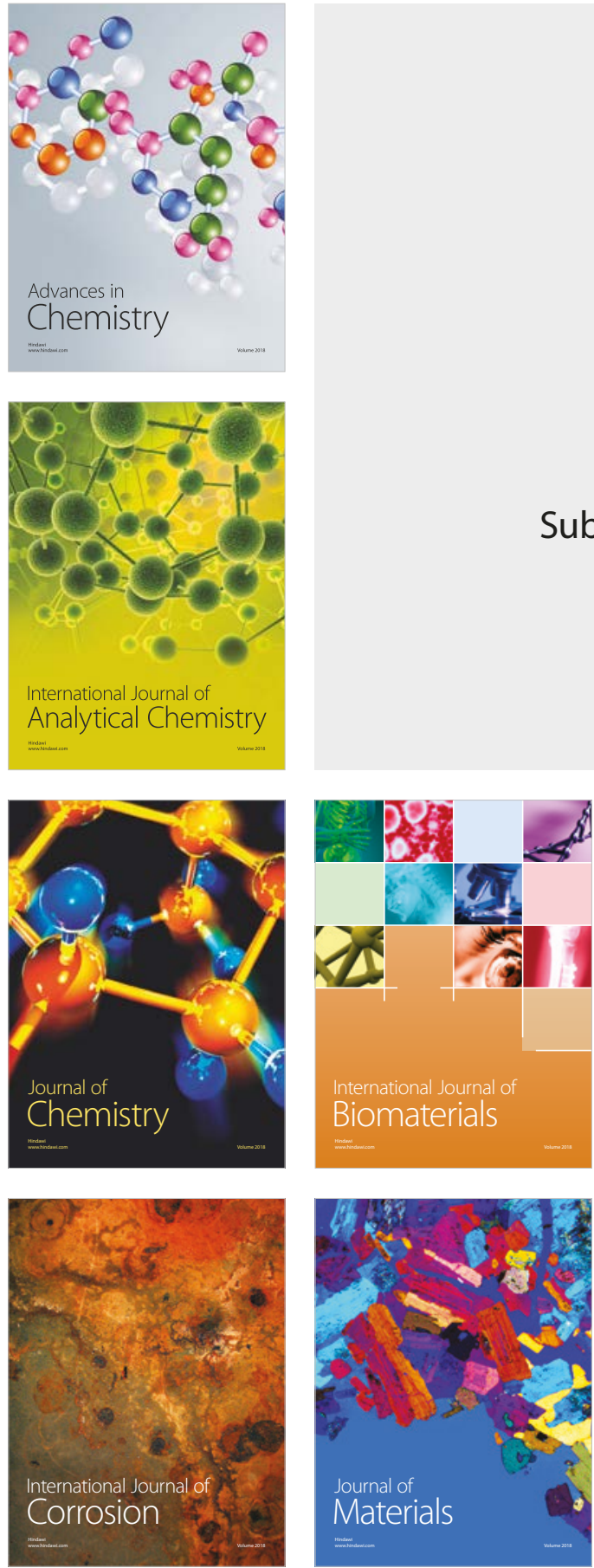

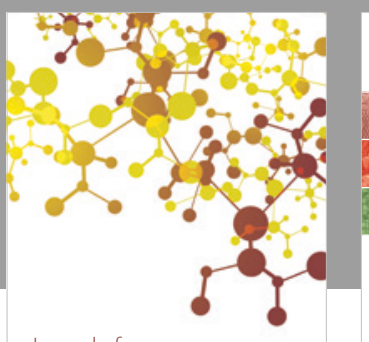

Journal of

Applied Chemistry
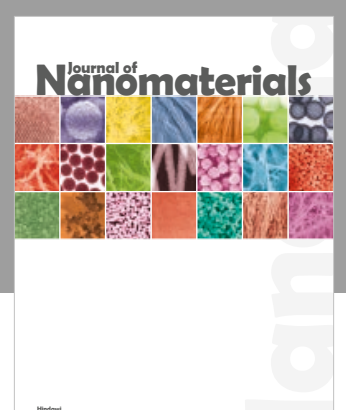

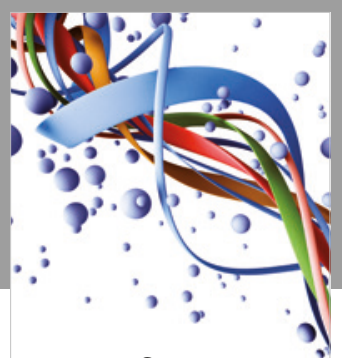

Scientifica

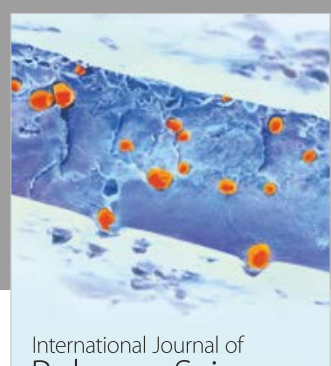

Polymer Science

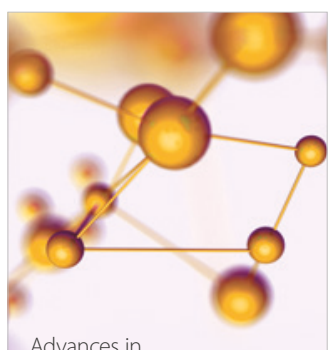

Physical Chemistry
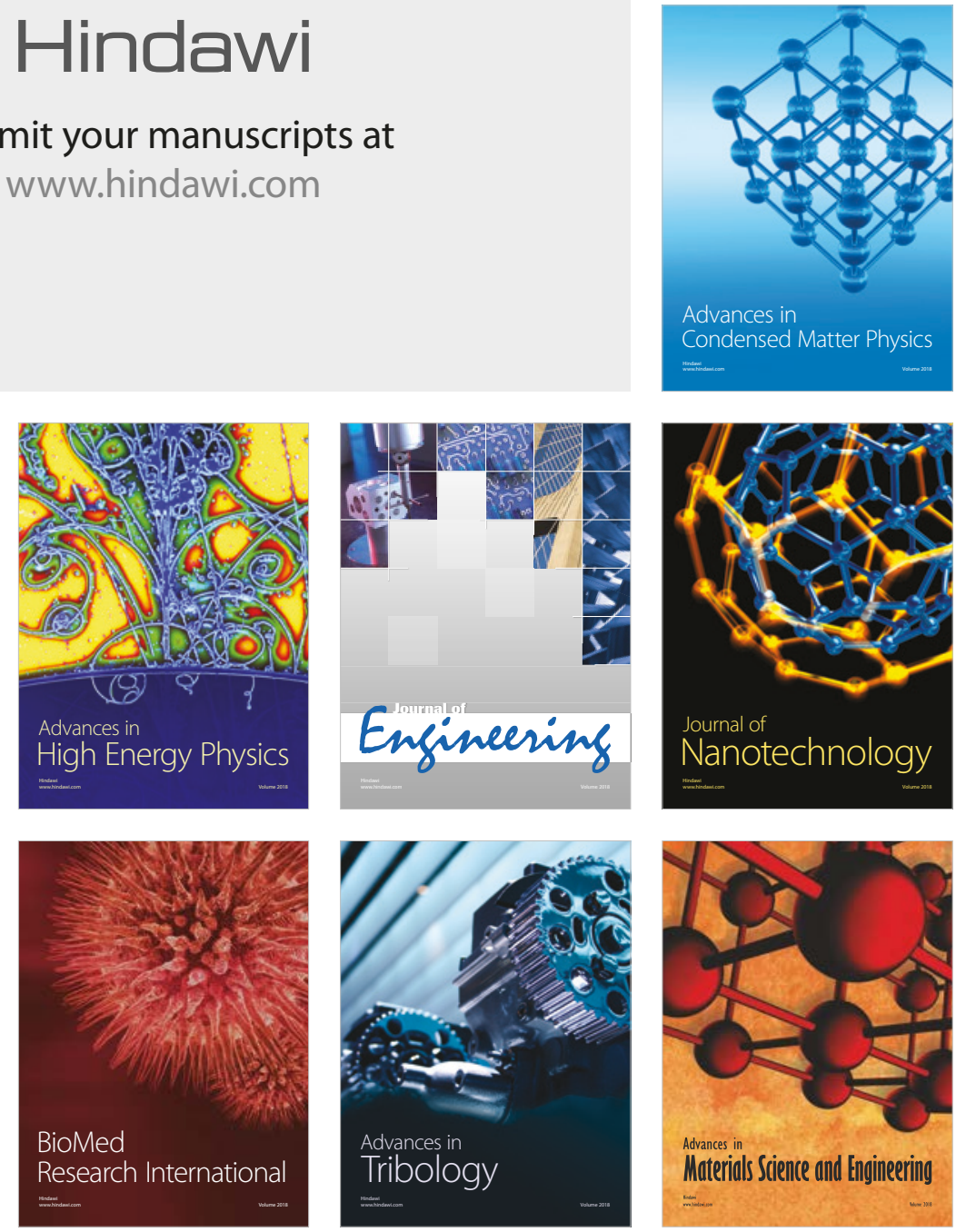